\title{
A new sign in the standard hip ultrasound image of the Graf method
}

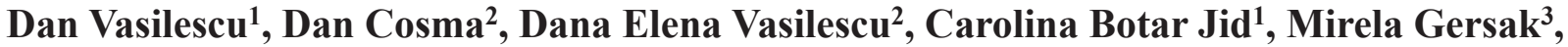 \\ Manuela Lenghel ${ }^{1}$, Sorin Marian Dudea ${ }^{1}$
}

${ }^{1}$ Radiology Desk, University of Medicine and Pharmacy "Iuliu Hațieganu”, ${ }^{2}$ Pediatric Surgery and Orthopedics, University of Medicine and Pharmacy "Iuliu Hațieganu”, ${ }^{3}$ Radiology Department, Emergency County Clinical Hospital, Cluj-Napoca, Romania

\begin{abstract}
Aim: The aim of the study was to assess the usefulness of obtaining a $90^{\circ}$ angle between the plane of the osteochondral plate and the surface of the transducer during standard hip ultrasonography according to Graf method. Material and methods: In this retrospective study 1078 patients (2156 hips) were included examined between 2008 and 2014 for developmental dysplasia of the hip (DDH) ultrasound screening. The patients were divided in two groups. Group I consisted of 402 patients examined between January 2008 and December 2011 using the standard Graf method. Group II consisted of 676 patients examined from January 2012 to December 2014 using the Graf method with an additional criterion: $90^{\circ}$ angle between the plane of the osteochondral plate and the surface of the transducer. Results: We found more dysplastic patients in group I comparing to group II: $55(13.7 \%)$ and $38(5.6 \%)$ respectively. The difference in the incidence of patients diagnosed with DDH was highly significant $(\mathrm{p}<0.001)$. The mean alpha angle value in group I was $65.31^{0}$, respectively $67.52^{0}$ for group II ( $\left.<<0.001\right)$. Conclusion: The new osteochondral plate sign has the potential to reduce the overdiagnosis of DDH and provide a better tailored approach to borderline hips.
\end{abstract}

Keywords: hip ultrasound, Graf method, osteochondral plate, DDH

\section{Introduction}

Developmental dysplasia of the hip (DDH) is a wellknown and studied condition, which can affect the hip joint throughout its development. Although numerous studies have focused on the issue of diagnosis, there is still no widespread consensus on the approach $[1,2]$. The literature presents many concepts ranging from purely clinical diagnosis to complex screening programs $[2,3]$.

One of the most popular hip ultrasound (US) diagnostic methods is the Graf method. Although the most commonly used, this method continues to generate discussion on the sensitivity and specificity, based on the rate of false positive or false negative diagnosis [4-6].

Received 02.04.2015 Accepted 5.05.2015

Med Ultrason

2015, Vol. 17, No 2, 206-210

Corresponding author: Dan Cosma

146-50 Viilor street

400347 Cluj-Napoca, Romania

Phone: +40745493041

E-mail: dicosma@gmail.com
The Graf method is based on obtaining the standard image of the hip joint. In this image it is mandatory to see the elements that certify the accuracy: lower limb (lower extremity of the bony acetabular roof), the plane of the iliac bone surface parallel to the surface of the transducer and the labrum. It is also necessary to correctly identify: the femoral head (with the ossification nucleus when present), perichondrium-periosteum junction, joint capsule (with a characteristic fold), greater trochanter, convexityconcavity deflection point and the osteochondral plate (visible in the inferior aspect of the femoral head) [7].

The main drawback incriminated is overdiagnosis leading to unnecessary treatment and increased health costs [8]. Some studies propose alternative US methods to the Graf technique and some countries and centers are adherent to these alternative methods [9-12].

Extensive studies have been conducted to assess the correlations between clinical and US diagnosis or between US and classic hip radiography [13]. The outcome of these complex studies was influenced by: long duration (the Graf method was last improved in 2006 [7]), the lack of a golden standard and the ethical conflict in using an 
irradiating technique such as radiography. The common criticisms of the Graf method are the high rates of overdiagnosis, interobserver variations in the measurements, and the dependence on the examiner's experience $[4,5,14,15]$.

The present study aims to assess the usefulness of an additional element of accuracy for the standard image of the Graf US method: a $90^{\circ}$ angle between the plane of the osteochondral plate and the surface of the transducer.

\section{Material and methods}

The study was conducted in the Radiology Department of the Emergency County Clinical Hospital ClujNapoca, by retrospective analysis of the database containing 2356 reports of the subjects presented for DDH assessment between January 2008 - December 2014. I n all cases hip US was performed according to the Graf method using Hitachi EUB 8500 US equipment with a 6.5-13 MHz linear transducer.

The study inclusion criteria were: subjects presenting for US hip screening for which the US diagnosis was either normal (Ia, Ib), immature hips, or mild dysplasia (IIa+, IIa- and IIb) according to Graf method hip staging criteria [7]. Patients with risk factors (family history, breach or transverse presentation, gemelarity, oligohydramnios, associated neuromuscular and musculoskeletal pathology: clubfoot, metatarsus adductus, torticollis, cerebral palsy) and with prior clinical examination or indication for hip US due to suspected DDH were excluded. Patients with severe DDH (IIc, D, IIIa, IIIb and IV) were also excluded.

From the total 2356 subjects examined between January 2008 and December 20141278 patients were excluded. The study group was limited to 1078 patients (2156 hips). The study group was divided into two groups, according to the period of time and the examination technique.

Group I consisted of patients examined between January 2008 and December 2011 (402 patients - 804 hips), who underwent US examination of the hip according to the classic standards of the Graf method.

Group II consisted of patients examined from January 2012 to December 2014 (676 patients - 1352 hips), who underwent US of the hip according to the Graf method with an additional quality criterion developed by our

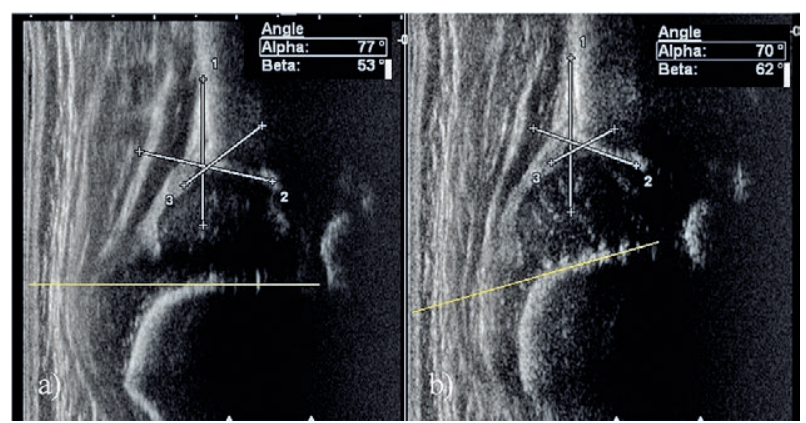

Fig 1. Standard ultrasound images of the same right hip. It can be seen the variation in the direction of the osteochondral plate yellow line: a) horizontal; b) superior oblique. On the right side of the images the angle measurements are included.

group, named the perpendicular osteochondral plate. Technique and description of the criterion under investigation: while obtaining the quality criteria of the Graf image, special attention was paid to angulate the transducer to a point where the osteochondral plate is visible on its width and the angle between the plane passing through the osteochondral plate and the surface of the transducer is $90^{\circ}$ (fig 1 ).

The institutional Ethics Committee approval was obtained and, due to the retrospective character of the study, written consent was waived.

Statistical analysis was performed using SPSS 13.0 for Windows software (SPSS Inc., Chicago, IL, USA) and the statistical tests were chosen according to the studied data type: descriptive statistics (Means, std. Dev, std. Error 95 $\%$ CI, Min, Max), Kolmogorov-Smirnov test, the MannWhitney $\mathrm{U}$ test and $\mathrm{Z}$ test. The Kolmogorov-Smirnov test was used to determine the normality of the distribution in the study groups. The Mann-Whitney U test was used to assess not normally distributed data (average alpha angle values in the two groups). The $\mathrm{Z}$ test was used to compare the two population groups proportions. $\mathrm{P}$ was considered statistically significant at a value $\mathrm{p}<0.05$ for Mann-Whitney $\mathrm{U}$ test, respectively $\mathrm{p}<0.01$ for the $\mathrm{Z}$ test.

\section{Results}

The demographic analysis of the two groups is presented in table I.

Table I. Comparative demographic data of group I and II

\begin{tabular}{lllllllll}
\hline & Total no. & Gender & \multicolumn{3}{c}{ Age (weeks) } \\
& & m & f & f:m ratio & Av. & Std. dev & Min & Max \\
\hline Group I & 402 & $156(38.7 \%)$ & $246(61.3 \%)$ & 1.57 & 12.1 & 6.86 & 2 \\
Group II & 676 & 300 & 376 & 1.25 & 9.9 & 5.35 & 2 \\
& & $(44.4 \%)$ & $(55.6 \%)$ & & & & & 40 \\
\hline
\end{tabular}

male, f- female 

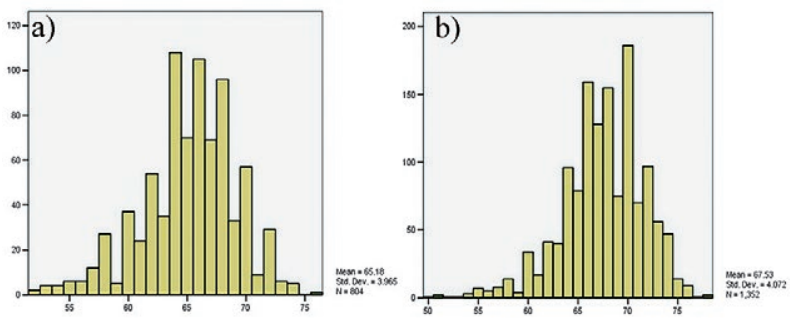

Fig 2. Non normal distribution of the alpha angle values in: a) group I, b) group II.

The distribution of the values of the alpha angle in the two groups is illustrated in figure 2.

The Kolmogorov-Smirnov test applied to both groups demonstrated non normal distribution of the data $(\mathrm{p}<0.05)$.

The alpha angle average of all hips from group I and II are shown in table II.

The Mann-Whitney U test showed significant difference between the means of the two groups $(p<0.001)$.

The alpha angle average was computed separately for the hips labeled as normal and for the abnormal hips, in both groups (table III).

Statistical analysis showed significant difference between the average alpha angle of normal labeled hips in groups I and II $(\mathrm{p}<0.001$, Mann-Whitney U test). There was no statistical difference between the average alpha angle of the abnormal hips in the two groups ( $\mathrm{p}=0.497$ ).

The incidence of abnormal alpha value diagnosed patients/hips was calculated in each group (table IV).
There was significant difference of incidence of the abnormal hips between the two groups ( $p<0.001$, Z-test).

The data shown in table III implies that the addition of the new quality criterion increases the average value of the obtained alpha angle by $2^{0}$. To assess this effect of the new quality criterion, a recalculation of all alpha angles in group I was performed by adding 2 units to the original value. Reclassification of the hips with the new values (group IR) provided an incidence of the abnormal labeled patients of $7.4 \%$ (30 patients). Comparing groups IR and II yielded no significant difference in the incidence of the abnormal patients ( $\mathrm{p}=0.115$, Z-test).

Evaluation of the obtained osteochondral plate sign in the archived images of the patients from group II showed $87 \%$ success rate.

\section{Discussions}

The introduction of hip US for DDH diagnosis was equally a great progress in pediatric clinical practice and created a distinct niche of uncontested clinical application of US examination. For more than 30 years the US Graf method is widely used for this purpose [16].

The main criticisms on the Graf method are represented by variability and examiner dependency. Graf described the quality criteria needed to produce interpretable and reproducible images [7]. One further criticism of the Graf method is the over-diagnosis.

This study aimed to analyze the impact of an additional quality criterion in obtaining the standard image.

Table II. Descriptive data analysis of the alpha angle for all subjects from group I and II

\begin{tabular}{lllllllll}
\hline & N & Mean & Std. Dev & Std. Error & \multicolumn{2}{c}{$\begin{array}{l}\text { 95\% Confidence Interval for Mean } \\
\text { Lower Bound }\end{array}$} & Min & Max \\
& & & & & & Lper Bound & \\
\hline Group I & 804 & 65.18 & 3.965 & 0.140 & 64.90 & 65.45 & 76 & 52 \\
Group II & 1352 & 67.53 & 4.072 & 0.111 & 67.31 & 67.75 & 50 & 78 \\
Total & 2156 & 66.65 & 4.189 & .0090 & 66.47 & 66.83 & 50 & 78 \\
\hline
\end{tabular}

Table III. Descriptive data analysis of the alpha angle for normal and abnormal subjects from group I and II

\begin{tabular}{llllllllll}
\hline & & No. & Mean & Std. Dev & Std. Error & \multicolumn{2}{c}{ 95\% Confidence Interval for Mean } & Min & Max \\
& & & & & & & Lower Bound & Upper Bound & \\
\hline Normal & Group I & 738 & 65.93 & 3.137 & 0.115 & 65.71 & 66.16 & 60 & 76 \\
& Group II & 1306 & 67.92 & 3.515 & 0.097 & 67.73 & 68.12 & 60 & 78 \\
& Total & 2044 & 67.21 & 3.516 & 0.078 & 67.05 & 67.36 & 60 & 78 \\
\multirow{5}{*}{ Abnormal } & Group I & 66 & 56.71 & 1.846 & 0.227 & 56.26 & 57.17 & 52 & 59 \\
& Group II & 46 & 56.26 & 2.245 & 0.331 & 55.59 & 56.93 & 50 & 59 \\
& Total & 112 & 56.53 & 2.022 & 0.191 & 56.15 & 56.91 & 50 & 59 \\
\hline
\end{tabular}

Table IV. Comparative incidence of normal and abnormal patients / hips in group I and II

\begin{tabular}{llll}
\hline & Total No. patients / hips & Normal patients / hips & Abnormal patients / hips \\
\hline Group I & $402 / 804$ & $347(86.3 \%) / 738(91.8 \%)$ & $55(13.7 \%) / 66(8.2 \%)$ \\
Group II & $676 / 1352$ & $638(94.4 \%) / 1306(96.6 \%)$ & $38(5.6 \%) / 46(3.4 \%)$ \\
\hline
\end{tabular}


For this purpose, the allotment of the subjects in one of the two patient groups based was on two considerations: the period of time and the method of investigation used. Although unequal, the chosen time intervals aimed at the inclusion of a sufficient number of patients to allow significant statistical studies. Since US is used as a screening method, only patients who were presented in department for this purpose were included in the study groups. This is the reason why patients presenting risk factors, with previous clinical exam or indication for hip US were excluded. Given the sporadic and unpredictable occurrence and the potential influence on the population average values, US diagnosed severe dysplasia (IIc, D, IIIa, IIIb and IV) cases were also excluded from the study. The final difference between the 2 groups consisted only of that for group I, the classic Graf method was applied and for group II the Graf method with the additional accuracy criterion was used. In evaluating the results, it was assumed nonvariable DDH incidence in the constant ethnic population over a few years as stated by Randall et al [1].

The demographic study revealed a difference in females/males ratio of 1.57:1 in group I compared with $1.25: 1$ in group II. This can be explained by the wider acceptance, in the last years, of universal US screening idea without taking into account the baby's gender. In the same context, better information on the need to perform hip US led to a decrease of the average age at first presentation in the second group, closer to the recommended age of 4-6 weeks [17].

Initial comparison between the two groups showed statistically significant differences in the alpha angle value of the patients labeled as normal. A $2^{0}$ higher average value of the patients from group II than group I was calculated $\left(67^{0}\right.$ vs $\left.65^{\circ}\right)$.

There was a statistically significant difference between the number of patients diagnosed having a less then $60^{\circ}$ alpha angle hips in the two groups: more normal hips diagnosed in group I as compared to group II. The $2^{0}$ angle difference may exert a major repercussion on borderline hips: type IIa-, IIa+ or IIb with alpha angle value close to $60^{\circ}$. This category of patients is the main cause for controversial literature data on the increased rate of over-diagnosis $[6,12,18,19]$.

The average alpha angle value of both normal and abnormal hips was calculated separately for both groups. For the normal labeled hips the difference of $2^{0}$ persisted and was validated by statistical analysis. There was no statistically significant difference between the average values of the abnormal hips in the two groups. These results infer that the perpendicular osteochondral plate sign may add an average value of $2^{0}$ at the measured alpha angle. Higher absolute value of the alpha angle may in its turn contribute to the reclassification of some of the borderline abnormal hips. As there was a significant difference in the percentage of abnormal hips in groups I and II (lower percentage in group II) and as this observation contradicts the original study assumption as stated above an attempt was made to validate the observation by reclassifying the abnormal hips in group I by adding $2^{0}$ to the initially measured value. In newly formed group IR, all hips from original group I and with alpha angles of $58^{\circ}$ and $59^{\circ}$ were relabeled as normal. From the 55 patients initially diagnosed as having abnormal hips, only 30 remained abnormal in group IR when using the new criterion. The newly obtained incidence of abnormal hips in group IR was $7.4 \%$, with no statistically significant difference from group II $(5.6 \%, \mathrm{p}>0.1)$. A large numeric study of Caucasians examined for DDH by hip US mentioned an incidence of $7.15 \%$ [20], similar to group II and IR from the present study (5.6\% and 7.4\%). The relatively increased number of abnormal cases does not mean an equal incidence of DDH. The incidence reported for group II and IR includes also immature hips.

Some limitations of the present study must be acknowledged. A limitation of the present study could be the success rate in obtaining the new criterion on the standard image. This is difficult or sometime impossible for very advanced mature hips when the osteochondral plate is located in the acoustic shadow generated by the ossified lateral part of the plate. In this situation the direction of the osteochondral plate is either perfect horizontal, or slightly oblique to inferior [7]. Therefore, when acquiring the standard image the osteochondral plate might be visible having a horizontal trajectory in an image immediately before obtaining the standard one. However, due to the constantly decreasing age at the presentation this was not considered a major limitation and as shown the success rate in group II was $87 \%$.

Due to the retrospective character of the study, interobserver and intraobserver variability assessment of the new criterion was not performed.

The study refers strictly to the US hip approach and not to the overall management of the case. Complete management of a DDH case is more complex and subject to further studies.

\section{Conclusions}

The present study suggest that the addition of a new quality criterion for the Graf method standard image may contribute to a better tailored approach to the US diagnosis of DDH by lowering the number of over-diagnosed cases. This criterion may be useful especially in cases with borderline values of the alpha angle. Further studies 
are mandated to confirm these observations and fully assess the clinical usefulness.

The presence of the osteochondral plate sign in the standard image of the Graf method has led to a decreased number of diagnosed DDH cases. This lowered case number remedies the over-diagnosis complaint of those who oppose US screening for DDH. As a consequence, it will reduce cases of unnecessary treatment, especially in patients diagnosed with mild or moderate DDH. Future studies on more meaningful numeric groups may confirm or refute our observation.

\section{Conflict of interest: none}

\section{References}

1. Loder RT, Skopelja EN. The epidemiology and demographics of hip dysplasia. ISRN Orthop 2011; 2011: 238607.

2. Roposch A, Graf R, Wright JG. Determining the reliability of the Graf classification for hip dysplasia. Clin Orthop Relat Res 2006; 447: 119-124.

3. Peterlein CD, Schuttler KF, Lakemeier S, et al. Reproducibility of different screening classifications in ultrasonography of the newborn hip. BMC Pediatr 2010; 10: 98.

4. Harcke HT. Screening newborns for developmental dysplsia of the hip. The role of sonography. Am J Roentgenol 1994; 162: 395-397.

5. Harcke HT. The role of ultrasound in the diagnostis and management of developmental dysplasia of the hip (DDH). Pediatr Radiol 1995; 3: 225-227.

6. Bracken J, Tran T, Ditchfield M. Developmental dysplasia of the hip: Controversies and current concepts. J Paediatr Child Health 2012; 48: 963-973.

7. Graf R. Hip Sonography: Diagnosis and Management of Infant Hip Dysplasia, Springer-Verlag Berlin Heidelberg 2006.

8. Shorter D, Hong T, Osborn DA. Cochrane Review: Screening programmes for developmental dysplasia of the hip in newborn infants. Evid Based Child Health 2013; 8: 11-54.

9. Falliner A, Schwinzer D, Hane HJ, Hedderich J, Hassenpflug J. Comparing ultrasound measurements of neonatal hips using the method of Graf and Terjesen. J Bone Joint Surg Br 2006; 88: 104-106.

10. Rakovac I, Tudor A, Sestan B, et al. New "L value" parameter simplifies and enhances hip ultrasound interpretation in the detection of developmental dysplasia of the hip. Int Orthop 2011; 35: 1523-1528.

11. Treguier C, Chapuis M, Branger B, et al. Pubo-femoral distance: an easy sonographic screening test to avoid late diagnosis of developmental dysplasia of the hip. Eur Radiol 2013; 23: 836-844.

12. Harcke HD, Grissom LE. Sonographic evaluation of the infant hip. Semin Ultrasound 1986; 7: 331-338.

13. Delaney LR, Karmazyn B. Developmental Dysplasia of the Hip: Background and the Utility of Ultrasound. Semin Ultrasound CT MRI 2011; 32: 151-156.

14. Graf R, Mohajer M, Plattner F. Hip sonography update. Quality-management, catastrophes - tips and tricks. Med Ultrason 2013; 15: 299-303.

15. Rosendahl K, Aslaksen A, Lie RT, Markestad T. Reliability of ultrasound in the early diagnosis of developmental dysplasia of the hip. Pediatr Radiol 1995; 25: 219-224.

16. Graf R. The diagnosis of congenital hip-joint dislocation by the ultra-sonic Combound treatment. Arch Orthop Trauma Surg 1980; 97: 117-133.

17. Riccabona M, Schweintzger G, Grill F, Graf R. Screening for the developmental hip dysplasia (DDH) - clinically or sonographically? Comments to the current discussion and proposals. Pediatr Radiol 2013; 43: 637-640.

18. Sibinski M, Adamczyk E, Higgs ZC, Synder M. Hip joint development in children with type IIb developmental dysplasia. Int Orthop 2012; 36: 1243-1246.

19. Omeroglu $\mathrm{H}$. Use of ultrasonography in the developmental dysplasia of the hip. J Child Orthop 2014; 8: 105-113.

20. Bialik V, Bialik GM, Blazer S, Sujov P, Wiener F, Berant M. Developmental dysplasia of the hip: a new approach to incidence. Pediatrics 1999; 103: 93-99. 